Artigo

\title{
Comitês de Mortalidade Infantil e Fetal: importância, finalidade e funcionamento
}

\author{
Child and Fetal Mortality Committees: \\ importance, purpose and operation
}

\section{Comités Infantil y de Mortalidad Fetal: importancia, finalidad y funcionamiento}

\section{Comités de Mortalité des Enfants et Fotal: importance, objectif et fonctionnement}

Mara Rúbia Campos Teixeira, ${ }^{1}$, Beatriz de Castro Magalhães ${ }^{2}$ e Grayce Alencar Albuquerque ${ }^{3}$

\footnotetext{
Enfermeira, especialista em Saúde da Família, Residente em Saúde da Família e comunidade e coordenadora do programa de Agentes Comunitários de Saúde na Secretaria de Saúde do município de Acopiara, CE, Brasil.

E-mail: mararct.enf@gmail.com

${ }^{2}$ Enfermeira, mestranda em enfermagem no Programa de Pós-Graduação em Enfermagem da Universidade Regional do Cariri, Crato, CE, Brasil.

E-mail: beatriz.castro022015@gmail.com

Doutora em Ciências da Saúde pela Faculdade de Medicina do ABC, Santo André, SP, Brasil. Professora Adjunta, Coordenadora do Observatório da Violência e dos Direitos Humanos da região do Cariri, docente no Curso de Enfermagem e nos Programas de PósGraduação em Enfermagem e em Saúde da Família da Universidade Regional do Cariri, Crato, CE, Brasil.

E-mail: geycyenf.ga@gmail.com
} 
Resumo

\section{Abstract}

O objetivo do artigo foi refletir sobre a atuação dos Comitês de Mortalidade Infantil e Fetal frente às ações implantadas para superar os desafios e determinantes que contribuem para um maior número de óbitos dessas populações. Trata-se de uma revisão narrativa da literatura que se deu por meio do levantamento de artigos e documentos oficiais referentes à operacionalização dos Comitês de Mortalidade Infantil e Fetal no Brasil. Os achados indicam que os Comitês de Mortalidade Infantil e Fetal devem ser, necessariamente, de forma efetiva, um pólo técnico/gerencial, cujas ações ou recomendações fluam com total respaldo das autoridades. Sobre sua atuação, os Comitês apontam dificuldades para reduzir os óbitos infantis e fetais, mediante obstáculos para acesso à saúde de qualidade, precarização das instituições de saúde e deficiências nos processos de trabalho das equipes multidisciplinares presentes nas redes de atenção. Conclui-se que há um quadro ainda preocupante no que se refere à ações resolutivas para a redução da mortalidade infantil e fetal, sendo a implantação dos Comitês de Mortalidade Infantil e Fetal, um avanço no sentido de promover meios de, a partir da compreensão dos fatores relacionados a óbitos evitáveis, implantar medidas efetivas para a sua redução.

Palavras-Chave: Mortalidade Infantil; Mortalidade Fetal; Vigilância em Saúde Pública; Gestão das Informações em Saúde; Gestão em Saúde.

The objective of the article was to reflect on the performance of the Infant and Fetal Mortality Committees in view of the actions implemented to overcome the challenges and determinants that contribute to a greater number of deaths among these populations. It is a narrative review of the literature that took place through the survey of articles and official documents regarding the operationalization of the Infant and Fetal Mortality Committees in Brazil. The findings indicate that the Infant and Fetal Mortality Committees must necessarily be effectively an technical / managerial hub, whose actions or recommendations flow with full support from the authorities. Regarding their performance, the Committees point out difficulties in reducing infant and fetal deaths, through obstacles to access quality health care, precarious health institutions and deficiencies in the work processes of the multidisciplinary teams present in the care networks. It is concluded that there is still a worrying picture regarding resolutive actions for the reduction of infant and fetal mortality, with the implantation of the Infant and Fetal Mortality Committees, an advance in the sense of promoting means of, from the understanding of the factors related to preventable deaths, implement effective measures to reduce them.

Keywords: Infantmortality; Fetal Mortality; Public Health Surveillance; Health Information Management; Health Management. 
Resumen

\section{Resumé}

El objetivo del artículo fue reflexionar sobre el desempeño de los Comités de Mortalidad Infantil y Fetal en cuanto a las acciones implementadas para superar los desafíos y determinantes que contribuyen a un mayor número de muertes entre estas poblaciones. Se trata de una revisión narrativa de la literatura que se realizó a través de la encuesta de artículos y documentos oficiales sobre la operacionalización de los Comités de Mortalidad Infantil y Fetal en Brasil. Los hallazgos indican que los Comités de Mortalidad Infantil y Fetal deben necesariamente ser efectivamente, un polo técnico / gerencial, cuyas acciones o recomendaciones fluyan con pleno apoyo de las autoridades. En cuanto a su desempeño, los Comités señalan dificultades en la reducción de muertes infantiles y fetales, a través de obstáculos para acceder a una atención de salud de calidad, instituciones de salud precarias y deficiencias en los procesos de trabajo de los equipos multidisciplinarios presentes en las redes de atención. Se concluye que aún existe un panorama preocupante en cuanto a acciones resolutivas para la reducción de la mortalidad infantil y fetal, con la implantación de los Comités de Mortalidad Infantil y Fetal, un avance en el sentido de promover medios de, desde la comprensión de los factores relacionados a las muertes evitables, implementar medidas para reducirlas.

Palabras clave: Mortalidad Infantil; Mortalidad Fetal; Vigilancia em Salud Pública; Gestión de la Información en Salud; Gestión en Salud.

L'objectif de l'article était de réfléchir sur la performance des Comités Mortalité Infantile et Fœtale concernant les actions mises en œuvre pour surmonter les défis et déterminants qui contribuent à un plus grand nombre de décès parmi ces populations. Il s'agit d'une revue narrative de la littérature qui a eu lieu à travers l'enquête d'articles et de documents officiels concernant l'opérationnalisation des comités de mortalité infantile et fœtale au Brésil. Les résultats indiquent que les comités de mortalité infantile et fœtale doivent nécessairement être effectivement, un pôle technique / managérial, dont les actions ou recommandations découlent du plein appui des autorités. Concernant leurs performances, les Comités soulignent les difficultés de réduction des décès infantiles et fotaux, à travers les obstacles à l'accès à des soins de santé de qualité, la précarité des établissements de santé et les carences dans les processus de travail des équipes multidisciplinaires présentes dans les réseaux de soins. On en conclut qu'il existe encore un tableau inquiétant concernant les actions résolutives de réduction de la mortalité infanto-fœtale, avec l'implantation des Comités de mortalité infantile et fœtale, une avancée dans le sens de la promotion des moyens de, à partir de la compréhension des facteurs liés pour éviter les décès, mettre en œuvre des mesures efficaces pour les réduire.

Mots Clés: Mortalité Infantile; Mortalité Foetale; Surveillance de la Santé Publique; Gestion de l'Information en Santé; Gestion de la Santé. 


\section{Introdução}

A redução da mortalidade infantil tem sido um processo em construção no Brasil, cujos resultados são frutos de políticas e ações que se efetivam através de serviços de qualidade ofertados durante a gestação, parto e nascimento. Apesar de ainda haver alguns desafios frente à operacionalização de políticas públicas nesse sentido, de acordo com o Instituto Brasileiro de Geografia e Estatística (IBGE), tem havido declínio na taxa de mortalidade infantil no Brasil (IBGE, 2019).

Entre 1940 e 2018, a mortalidade infantil no Brasil apresentou declínio da ordem de 91,6\%, enquanto que a mortalidade entre 01 a 04 anos de idade teve redução de $97,2 \%$. Neste período foram poupadas aproximadamente 134 vidas de crianças menores de 01 ano para cada mil nascidas vivas (IBGE, 2019).

De um modo geral, a mortalidade infantil decorre não somente de um fator isolado, mas da interação de fatores biológicos, sociais, culturais e de falhas do sistema de saúde, visto que esses influenciam o desfecho "óbito", tanto para o feto, como para a criança em seus primeiros dias de vida. Embora a mortalidade infantil possua causas que foram historicamente negligenciadas pelos serviços de saúde, seja pela ausência de avaliações e análises sistemáticas de sua ocorrência, bem como, pela ausência de investimentos específicos a sua redução; nota-se que tais causas são, em sua maioria, potencialmente evitáveis (Brasil, 2009a).

Nesse contexto, vale destacar que a baixa qualidade na assistência obstétrica e neonatal se associa à ocorrência dos óbitos fetais e neonatais, sendo esta condição agravada pela intersecção dos determinantes sociais de saúde, como idade materna avançada, baixo nível socioeconômico, falta de apoio gestacional, baixa educação em saúde e localização geográfica; os quais são particularidades que devem ser abordadas durante a assistência em saúde (Silva et al., 2019).

Além disso, ações inexistentes e/ou inadequadas realizadas especificamente durante o trabalho de parto e parto também mostram significantes impactos na mortalidade fetal e neonatal. No estudo de Martins et al. (2013), realizado em Minas Gerais, com 253 casos de mortalidade infantil investigados, ocorridos após a admissão da gestante no hospital, identificou-se que em 65,6\% dos casos houve falha na assistência. Dessa porcentagem, $22 \%$ esteve relacionado a admissão, com destaque para a avaliação incompleta; $91,6 \%$ relacionado ao acompanhamento da gestante $(91,6 \%)$, destacando-se a frequência do intervalo de avaliação materno-fetal superior ao recomendado e a demora no manejo das complicações do trabalho de parto e parto; $11,5 \%$ associada à relação profissional/cliente insatisfatória, com queixas de atendimento desumanizado e falta de informação e $15 \%$ relativas à dificuldade de acesso da gestante à maternidade.

Assim, é fundamental destacar a relevância da vigilância do óbito infantil, a qual se destaca como uma estratégia significativa para a manutenção da redução das taxas de mortalidade infantil e tem por objetivo incorporar o uso de informações qualificadas para o planejamento e avaliação de políticas públicas e ações em saúde. Assim, com ênfase no alcance desse objetivo, tem-se o lançamento do Projeto de Redução da Mortalidade Infantil em 1995, que impulsionou a implantação de Comitês de Mortalidade Infantil e Fetal no país (Brasil, 2000). Além disso, em 2010, foi estabelecido pelo Ministério da Saúde, a vigilância do óbito infantil e fetal e a obrigatoriedade da investigação dos óbitos infantis e fetais no país (Brasil, 2010). 
Essas são políticas importantes para a superação dos desafios frente a redução da mortalidade infantil, entre eles, aquele que pode ser considerado um dos maiores na busca pela redução da taxa de mortalidade infantil no Brasil, que são as diferenças existentes entre as regiões brasileiras, mesmo existindo esforços para universalizar o acesso aos serviços e às ações de saúde e políticas implementadas por diversos setores do governo (Frias \&Navarro, 2013).

Ao considerar que um número significativo de óbitos infantis possui causas evitáveis, vislumbrase como necessário uma avaliação das condições de vida e saúde da população, provendo meios para que sejam implantadas intervenções em prol da superação dessas causas. Neste contexto, é importante que se destaque o papel dos comitês de prevenção do óbito infantil e fetal, para promover visibilidade ao fenômeno, realizar o acompanhamento e monitoramento dos óbitos infantis e fetais e propor intervenções para a sua redução (Brasil, 2009a).

Assim, diante da importância da vigilância dos óbitos infantis e fetais, tem-se como objetivo refletir sobre a atuação dos Comitês de Mortalidade Infantil e Fetal em relação às ações implantadas como forma de superar os desafios e determinantes que contribuem para um maior número de óbitos dessas populações.

A motivação para a exploração desta temática surge da sua importância por se tratar de tema cuja relevância é visível, dado que, apesar de o Brasil, em um panorama geral, mostrar redução nos índices de mortalidade infantil e fetal, ainda existem disparidades regionais que se acentuam e que colocam em risco de óbito este grupo populacional.

\section{Referencial Teórico}

Trata-se de uma revisão narrativa de literatura. Destaca-se a relevância deste tipo de estudo, com base em Elias et al., (2012), sendo possível atualizar-se sobre os achados em relação à busca de respostas a um determinado problema, construindo assim, um conhecimento maior sobre o assunto.

A revisão narrativa de literatura objetiva trazer uma síntese de resultados de pesquisas/conteúdo acerca de um tema em específico. A partir deste tipo de revisão, analisa-se as produções bibliográficas em determinada área do conhecimento com base em um tema exclusivo, apresentando novas ideias, métodos e referências que têm recebido maior ou menor ênfase na literatura selecionada (Elias et al., 2012).

As bases de dados utilizadas para esse levantamento foram Medical Line (MEDLINE), Literatura Latino-Americana e do Caribe em Ciências da Saúde (LILACS) e portal da Scientific Eletronic Library Online (SCIELO), utilizando-se os descritores Mortalidade infantil, Mortalidade Fetal e Vigilância em Saúde Pública, a partir dos quais originou-se 16 resultados. Não houve recorte temporal delimitado, dado o caráter flexível da revisão narrativa. Definiu-se como critérios de inclusão para seleção destes materiais àqueles disponíveis on-line, completos, em português e com temática relacionada à pesquisa, e dessa forma, foram incluídos oito artigos na amostra final.

Os resultados foram analisados e discutidos com base nos estudos pertinentes à temática, bem como, a partir dos documentos oficiais do Ministério da Saúde, Brasil. 
A elaboração deste trabalho implicou em leitura atentiva da bibliografia identificada, em que ocorreu leitura inicial dos títulos, posteriormente resumos e para àqueles que correspondiam aos objetivos da revisão, leitura do material na íntegra. As publicações selecionadas foram lidas e as informações obtidas analisadas, de forma descritiva, passando a fazer parte do corpo da revisão. Na análise descritiva dos dados da literatura, foram observados pontos em comuns e controversos que sustentam cientificamente os resultados.

\section{Resultados e Discussão}

A maioria dos estudos identificados foram realizados nas regiões Sul e Sudeste do Brasil (n=07), com maior predomínio de estudos documentais $(n=05)$, como se pode observar no quadro 01 abaixo.

Quadro 01 - Quadro sinóptico dos resultados da pesquisa. Brasil, 2021.

\begin{tabular}{|c|c|c|c|c|c|}
\hline Título & $\begin{array}{l}\text { Autores } \\
\text { e ano }\end{array}$ & Objetivo & $\begin{array}{l}\text { País, região e } \\
\text { Estado em } \\
\text { que foi } \\
\text { desenvolvido }\end{array}$ & $\begin{array}{c}\text { Desenho } \\
\text { metodológico }\end{array}$ & Resultados \\
\hline $\begin{array}{l}\text { Fatores de risco } \\
\text { relacionados à } \\
\text { mortalidade } \\
\text { fetal }\end{array}$ & $\begin{array}{l}\text { Klein, J. } \\
\text { M. et } \\
\text { al., } \\
2012 \text {. }\end{array}$ & $\begin{array}{l}\text { Analisar os fatores } \text { de } \\
\text { risco associados } \\
\text { mortalidade fetal }\end{array}$ & $\begin{array}{l}\text { Brasil, } \\
\text { Região Sul, } \\
\text { Rio grande do } \\
\text { Sul }\end{array}$ & $\begin{array}{l}\text { Estudo } \\
\text { documental de } \\
\text { caso-controle. }\end{array}$ & $\begin{array}{l}\text { A taxa de mortalidade fetal correspondeu a } \\
16,8 / 1.000 \text { nascimentos vivos. Depois da } \\
\text { análise multivariada, as variáveis que } \\
\text { persistiram significativamente associadas ao } \\
\text { óbito fetal foram: presença de } \\
\text { malformações, número de consultas durante } \\
\text { o pré-natal inferior a seis, síndromes } \\
\text { hipertensivas, menos do que oito anos de } \\
\text { estudo e natimortalidade prévia. }\end{array}$ \\
\hline $\begin{array}{l}\text { Mortalidade } \\
\text { infantil: } \\
\text { principais } \\
\text { causas evitáveis } \\
\text { na região } \\
\text { Centro de } \\
\text { Minas Gerais, } \\
\text { 1999-2011 }\end{array}$ & $\begin{array}{l}\text { Lisboa, } \\
\text { L. et al., } \\
2012 \text {. }\end{array}$ & $\begin{array}{l}\text { Descrever a evolução da } \\
\text { mortalidade infantil } \\
\text { evitável na região Centro } \\
\text { do estado de Minas } \\
\text { Gerais, Brasil, no período } \\
\text { 1999-2011. }\end{array}$ & $\begin{array}{l}\text { Brasil, } \\
\text { Região } \\
\text { Sudeste, } \\
\text { Minas Gerais }\end{array}$ & $\begin{array}{l}\text { Estudo } \\
\text { documental, } \\
\text { descritivo. }\end{array}$ & $\begin{array}{l}\text { A Taxa de mortalidade infantil por causas } \\
\text { evitáveis diminuiu de } 14,5 \text { para } 7,7 / 1000 \\
\text { nascidos vivos no período, com maior } \\
\text { redução no subgrupo 'Ações de Promoção à } \\
\text { Saúde' e menor no subgrupo 'Atenção à } \\
\text { Mulher na Gestação'; em 2009-2011, } \\
\text { entretanto, } 65 \% \text { dos óbitos infantis ainda } \\
\text { foram classificados como evitáveis. }\end{array}$ \\
\hline $\begin{array}{l}\text { Desigualdades } \\
\text { sócio-espaciais } \\
\text { da adequação } \\
\text { das } \\
\text { informações de } \\
\text { nascimentos e } \\
\text { óbitos do } \\
\text { Ministério da } \\
\text { Saúde, Brasil, } \\
2000-2002\end{array}$ & $\begin{array}{l}\text { Andrade } \\
\text {, C. L. } \\
\text { T. e } \\
\text { Szwarc } \\
\text { wald, } \\
\text { C.L., } \\
\text { 2007. }\end{array}$ & $\begin{array}{l}\text { Analisar as desigualdades } \\
\text { sócio-esapaciais da } \\
\text { adequação ras das } \\
\text { informaçes } \\
\text { nascimentos (SINASC) e } \\
\text { óbitos de } \\
\text { Ministério da Saúde para o } \\
\text { cálculo da mortalidade } \\
\text { infantil por município. }\end{array}$ & $\begin{array}{l}\text { Brasil, todas } \\
\text { as regiões }\end{array}$ & $\begin{array}{l}\text { Estudo } \\
\text { documental, } \\
\text { descritivo. }\end{array}$ & $\begin{array}{l}\text { Os resultados mostram desigualdades sócio- } \\
\text { espaciais importantes: o percentual de } \\
\text { adequação é maior no Centro-Sul e entre os } \\
\text { municípios de maior porte populacional. Em } \\
\text { relação aos três aspectos estudados, o } \\
\text { Sistema de Informação de Nascidos Vivos } \\
\text { teve a melhor avaliação. Sobre o Sistema de } \\
\text { Informação sobre Mortalidade, é preciso } \\
\text { reduzir a subnotificação e melhorar a } \\
\text { qualidade do preenchimento da causa de } \\
\text { óbito, para que as informações sustentem a } \\
\text { orientação dos programas de saúde voltados } \\
\text { para a redução das iniqüidades da } \\
\text { mortalidade infantil no Brasil. }\end{array}$ \\
\hline $\begin{array}{l}\text { Atividades de e } \\
\text { Atividades de } \\
\text { extensão }\end{array}$ & $\begin{array}{l}\text { Mathias, } \\
\text { T. A. F. } \\
\text { et al., }\end{array}$ & $\begin{array}{lr}\text { Descrever atividades } \\
\text { desenvolvidas em Projeto } \\
\text { de Extensão Universitária, }\end{array}$ & $\begin{array}{l}\text { Brasil, } \\
\text { Região Sul, } \\
\text { Paraná. }\end{array}$ & $\begin{array}{l}\text { Relato de } \\
\text { experiência, } \\
\text { descritivo. }\end{array}$ & $\begin{array}{l}\text { A atuação do Comitê e o contato com os } \\
\text { sistemas de informação vêm contribuindo } \\
\text { junto à equipe participante do projeto de }\end{array}$ \\
\hline
\end{tabular}




\begin{tabular}{|c|c|c|c|c|c|}
\hline \begin{tabular}{lr}
\multicolumn{2}{l}{ universitária em } \\
comitê & de \\
extensão & \\
universitária em \\
comitê & de \\
prevenção & de \\
mortalidade & \\
infantil & e \\
estatísticas & de \\
saúde & de \\
prevenção & de \\
mortalidade & \\
infantil & e \\
estatísticas & de \\
saúde & \\
\end{tabular} & 2009. & 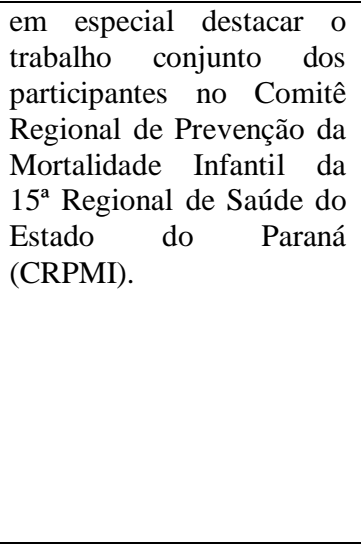 & & & $\begin{array}{l}\text { extensão, a compreensão do funcionamento, } \\
\text { da lógica e importância das informações em } \\
\text { saúde e de cada banco de dados que } \\
\text { compõem os Sistemas de Informação do } \\
\text { Sistema Único de Saúde. }\end{array}$ \\
\hline $\begin{array}{l}\text { Mortalidade } \\
\text { infantil e } \\
\text { desigualdade } \\
\text { social: análise } \\
\text { fundamentada } \\
\text { na Bioética de } \\
\text { Intervenção }\end{array}$ & $\begin{array}{l}\text { Bernardi } \\
\text { no, L. } \\
\text { C. S et } \\
\text { al., } \\
2015 \text {. }\end{array}$ & $\begin{array}{l}\text { Correlacionar Coeficiente } \\
\text { de Mortalidade Infantil } \\
\text { (CMI) de municípios de } \\
\text { Minas Gerais com } \\
\text { indicadores } \\
\text { socioeconômicos, } \\
\text { serviços e investimentos } \\
\text { em saúde, mediante } \\
\text { fundamentação na bioética } \\
\text { de intervenção. Trata-se } \\
\text { de estudo de delineamento } \\
\text { ecológico de grupos } \\
\text { múltiplos. }\end{array}$ & $\begin{array}{l}\text { Brasil, } \\
\text { Região } \\
\text { Sudeste, } \\
\text { Minas Gerais }\end{array}$ & $\begin{array}{l}\text { Estudo } \\
\text { documental, } \\
\text { ecológico, } \\
\text { correlacional. }\end{array}$ & $\begin{array}{l}\text { O maior Coeficiente de Mortalidade Infantil } \\
\text { correlacionou-se com os piores indicadores } \\
\text { socioeconômicos e de investimento em } \\
\text { saúde, sugerindo determinação social e } \\
\text { influência do contexto nos óbitos infantis. }\end{array}$ \\
\hline $\begin{array}{l}\text { Assistência de } \\
\text { enfermagem no } \\
\text { pré-natal e } \\
\text { evitabilidade de } \\
\text { óbitos neonatais }\end{array}$ & $\begin{array}{l}\text { Brandão } \\
\text {, I. C. } \\
\text { A., } \\
\text { Godeiro, } \\
\text { A. L. S. } \\
\text { e } \\
\text { Monteir } \\
\text { o, A. I., } \\
\text { 2012. }\end{array}$ & $\begin{array}{l}\text { Discutir acerca } \text { das } \\
\text { principais causas de óbitos } \\
\text { neonatais reduzíveis por } \\
\text { adequada atenção à } \\
\text { mulher na gestação, no } \\
\text { município de Natal, no } \\
\text { Estado do Rio Grande do } \\
\text { Norte, no período de } 2008 \\
\text { a } 2010 \text {, relacionando-as à } \\
\text { assistência de enfermagem } \\
\text { prestada à gestante } \\
\text { durante o pré-natal. }\end{array}$ & $\begin{array}{l}\text { Brasil, } \\
\text { Região } \\
\text { Nordeste, Rio } \\
\text { Grande do } \\
\text { Norte. }\end{array}$ & $\begin{array}{l}\text { Estudo } \\
\text { documental, } \\
\text { epidemiológic } \\
\text { o, descritivo e } \\
\text { retrospectivo. }\end{array}$ & $\begin{array}{l}\text { Do total de } 344 \text { óbitos neonatais } 78 \text {, foram } \\
\text { classificados como reduzíveis por adequada } \\
\text { atenção à mulher na gestação. }\end{array}$ \\
\hline $\begin{array}{l}\text { O processo de } \\
\text { análise da } \\
\text { evitabilidade } \\
\text { dos casos de } \\
\text { óbito infantil e } \\
\text { fetal: estudo de } \\
\text { caso único }\end{array}$ & $\begin{array}{l}\text { Ruoff, } \\
\text { A. B., } \\
\text { Andrade } \\
\text { S. R. e } \\
\text { Pocoli, } \\
\text { T., } \\
2018 .\end{array}$ & $\begin{array}{l}\text { Evidenciar o processo de } \\
\text { análise da evitabilidade } \\
\text { dos casos de óbito infantil } \\
\text { e fetal realizado por um } \\
\text { Comitê municipal de uma } \\
\text { capital do Sul do Brasil. }\end{array}$ & $\begin{array}{l}\text { Brasil, } \\
\text { Região Sul, } \\
\text { Santa } \\
\text { Catarina. }\end{array}$ & $\begin{array}{l}\text { Estudo de } \\
\text { caso, } \\
\text { descritivo. }\end{array}$ & $\begin{array}{l}\text { O resumo do caso de óbito infantil e fetal } \\
\text { mostra-se como um instrumento de } \\
\text { discussão, evidenciando os fatores que } \\
\text { contribuem para a ocorrência do óbito } \\
\text { infantil e fetal e as situações que } \\
\text { desencadeiam os óbitos, o que corrobora à } \\
\text { análise do caso de óbito na perspectiva da } \\
\text { evitabilidade. }\end{array}$ \\
\hline $\begin{array}{l}\text { O processo de } \\
\text { implantação } \\
\text { dos comitês de } \\
\text { investigação do } \\
\text { óbito infantil no } \\
\text { Estado de São } \\
\text { Paulo }\end{array}$ & $\begin{array}{l}\text { Venânci } \\
\text { o, S. I. e } \\
\text { Paiva, } \\
\text { R. }\end{array}$ & $\begin{array}{l}\text { Avaliar a implantação dos } \\
\text { Comitês de Investigação } \\
\text { do Óbito Infantil (CIOI) } \\
\text { no Estado de São Paulo } \\
\text { (SP). }\end{array}$ & $\begin{array}{l}\text { Brasil, } \\
\text { Região } \\
\text { Sudeste, São } \\
\text { Paulo. }\end{array}$ & $\begin{array}{lr}\text { Estudo } & \text { de } \\
\text { campo } & \\
\text { descritivo } & \text { e } \\
\text { estudo } & \text { de } \\
\text { caso. } & \end{array}$ & $\begin{array}{l}\text { Em } 87 \% \text { das Diretorias Regionais de Saúde } \\
\text { e 53\% dos municípios existiam Comitê de } \\
\text { Investigação do Óbito Infantil. As principais } \\
\text { dificuldades, foram: falta de estrutura } \\
\text { material e administrativa; atividades } \\
\text { conduzidas por iniciativas isoladas de } \\
\text { pessoas ou pequenos grupos; falta de } \\
\text { respaldo dos níveis superiores de hierarquia; } \\
\text { ações não contempladas nas instâncias de } \\
\text { planejamento; ausência de coordenação, } \\
\text { apoio e articulação de estratégias de redução } \\
\text { da mortalidade infantil em nível estadual. }\end{array}$ \\
\hline
\end{tabular}

Fonte: Dados da pesquisa. 
A taxa de mortalidade infantil encontra-se intrinsecamente relacionada com a situação de saúde e condições de vida da população, indicando que fragilidades existentes nas condições socioeconômicas, no desenvolvimento de políticas sociais e de saúde, em que se inclui o acesso e qualidade da assistência (Frias \& Navarro, 2013) repercute diretamente no óbito das crianças.

Tendo isso em vista, os dados sobre natimortos representam um conhecimento fundamental que permite avaliar as informações acerca das condições de saúde, assistência pré-natal e parto de uma determinada região. Mesmo apresentando uma redução, o número de óbitos fetais destaca o insucesso do processo gestacional, sendo um indicador importante para se avaliar a qualidade da assistência à saúde materno-infantil (Klein et al., 2012).

Em uma análise crítica, o próprio Ministério da Saúde no Brasil, reconhece que existem persistentes e perceptíveis desigualdades regionais e intraurbanas que devem ser superadas, pois reverberam em uma maior concentração dos óbitos na população mais pobre e iniquidades relacionadas a grupos sociais específicos (Brasil, 2009a), que em sua maioria, possuem dificuldades para acesso aos serviços de saúde, a exemplo do acompanhamento gestacional.

De fato, um fator a ser considerado diz respeito às consultas pré-natal. $\mathrm{O}$ número de consultas realizadas durante o pré-natal está diretamente relacionado à melhores indicadores de saúde maternoinfantil. Existem evidências de que a assistência pré-natal rotineira previne a morbimortalidade materna e perinatal, pois permite detecção e tratamento oportuno de afecções, além de reduzir fatores de risco que trazem complicações para a saúde da mulher e do bebê (Domingues et al., 2012). Assim, garantir acesso aos serviços, monitorar indicadores de assistência e de mortalidade são condições essenciais para enfrentamento do problema.

Em estudo de Mario et al. (2019) sobre a qualidade do pré-natal no Brasil, com dados de 1851 mulheres provenientes da Pesquisa Nacional de Saúde de 2013, foi identificado que o pré-natal adequado esteve presente em $80,6 \%$ dos casos, com maior prevalência entre as mulheres de cor branca $(87,7 \%)$ e que realizaram o pré-natal na rede privada $(92,7 \%)$. Além disso, a região Norte apresentou as menores frequências de pré-natal adequado, enquanto a região Sudeste as maiores, revelando que apesar da ampla cobertura, o pré-natal no Brasil ainda apresenta iniquidades e baixa qualidade no atendimento, especialmente sob a intersecção de raça e classe, em que mulheres não brancas e de regiões mais pobres do país encontram-se em desvantagem. Tais dados fazem refletir sobre os riscos elevados de óbito perinatal para essas mulheres.

Em relação a quantidade de pré-natais realizados no Brasil, a pesquisa de Tomasi et al., (2017), realizado com 6.125 mulheres que fizeram pré-natal na última gravidez entre 2012 e 2013, identificou que $89,1 \%$ realizaram seis ou mais consultas, com predomínio entre as mulheres de mais idade, brancas, de renda mais alta, moradoras na Região Sul e em municípios de menor porte.

Mediante esses estudos que destacam vulnerabilidades sob a intersecção de gênero, classe e raça, é importante ressaltar que durante muito tempo, mulheres e crianças não eram objeto de estudo da medicina. Após muita luta, principalmente do movimento feminista, passou-se a se pensar em programas que contemplasse essas populações, porém, ainda de forma integrada, como se as mulheres e as crianças apresentassem as mesmas necessidades. Dessa forma, a saúde da mulher foi se limitando ao aspecto reprodutivo, sob forte influência da medicalização dos corpos femininos (Silva et al., 2013).

No Brasil, somente a partir da década de 80, com a intensificação das lutas feministas, a Reforma Sanitária e a redemocratização do país, os diretos sexuais e reprodutivos passaram a ser pautas debatidas nas conferências nacionais de saúde, culminando na abordagem política, econômica, cultural e social do conceito de saúde e, posterior introdução da categoria gênero como instrumento útil para compreensão da situação da mulher na sociedade (Silva et al 2013), o que possui impacto também na 
saúde da criança por considerar as singularidades maternas que podem colocar em risco ou em proteção o desenvolvimento saudável de uma gestação, parto e pós-parto.

Apesar da evolução ocasionada pelos movimentos feminista e sanitário, ainda se percebe forte influência da maternidade compulsória, fruto de uma cultura que limitou, por muito tempo, a mulher ao seu aspecto reprodutivo, ditando o papel da mulher como indissociável à maternidade, além de projetar o estereótipo de mãe perfeita, a qual não pode demonstrar fragilidade, pois é de sua natureza (culturalmente) ser mãe (Lopez-Barreyro, 2017). Dessa forma, a experiência da maternidade se apresenta como experiência única e rodeada de significados, de esperanças e de promessas (Munozet al., 2013), o que aliado a uma atenção centrada predominantemente no modelo biologicista (Fertonani et al., 2015), desconsidera as singularidades maternas e os determinantes sociais de saúde, como gênero, raça e classe, implicando em maiores vulnerabilidades e infligindo os direitos sexuais e reprodutivos da mulher.

Além disso, ainda é válido notar que as desigualdades de gênero repercutem na disparidade econômica, uma vez que a divisão sexual do trabalho incute à mulher uma dupla carga de trabalho, marcado pelo trabalho doméstico não remunerado e pelo trabalho produtivo pouco valorizado e com remuneração muitas vezes mais baixa que para homens desempenhando as mesmas funções (Garcia, 2019). Além disso, as mulheres estão associadas a profissões consideradas "típicas de mulher", onde são geralmente mal pagas, sendo assim, minoria em áreas que pagam melhor (Garcia, 2019).

Assim, as mulheres estão mais propensas a integrarem o perfil de baixa renda e terem vulnerabilidades socioeconômicas que impactam na gestação, parto e vida pós parto da díade mãebebê, intensificando o risco para mortalidade infantil, principalmente considerando que muitas mulheres exercem maternidade solo (Galvão, 2020).

Nesse sentido, destaca-se que a mãe em condição de pobreza enfrenta sua maternidade com fragilidades, em grande parte derivadas da precariedade da sua existência, a saber: a chefia feminina da família, o subemprego (quando existe) e complicações no processo da gravidez, parto e puerpério (Munoz et al., 2013). Isso faz refletir sobre a intensificação das vulnerabilidades socioeconômicas das mulheres em face aos últimos governos (Temer e Bolsonaro), os quais demonstram maior desvalorização do salário mínimo, tendo por resultado a fragilização do salário mínimo como instrumento de redução das desigualdades sociais (Freitas, 2019).

Nesse sentido, é importante considerar os determinantes sociais imbricados à condição do ser mulher e a intersecção de raça e classe, bem como, a análise das vulnerabilidades com base na macroeconomia do Brasil, que repercutem diretamente em riscos para a saúde materna-infantil, podendo reverberar não somente na mortalidade materna, mas também na infantil.

Lisboa et al., (2015) tomando como referência alguns achados na literatura, observam que as medidas de mortalidade têm sua utilização de forma intencional, sendo usadas como indicadores da situação de saúde, como também, na avaliação e planejamento de políticas e programas de saúde. Citando algumas medidas, as autoras elencam a taxa de mortalidade infantil como uma das mais aceitas para mensurar o estado de saúde da população, considerando que sua referência está relacionada a mortes precoces, e em muitos casos, evitáveis.

De acordo com Andrade e Szwarcwald (2007) é fundamental o entendimento da importância do indicador de mortalidade infantil, que tem entre suas principais utilidades, proporcionar que seja revelada a situação de saúde do indivíduo ou de uma população, permitindo a avaliação do risco do evento ou agravo à saúde, auxiliando na mensuração das atividades de atenção à saúde e facilitando que sejam estabelecidos objetivos e metas na área materno infantil.

Este indicador é um parâmetro que tradicionalmente é medido pelo coeficiente de mortalidade infantil, e por meio dele, se faz a avaliação da situação de saúde das populações em regiões distintas. 
Contudo, mesmo sendo um indicador utilizado mundialmente, não se pode ainda afirmar que os dados sejam suficientemente consistentes no que diz respeito à cobertura e qualidade, principalmente, quando se trata de países em desenvolvimento (Andrade \& Szwarcwald, 2007).

Além do conhecimento e monitoramento deste indicador, importante se faz enfatizar pesquisas sobre evitabilidade dos óbitos, cujas causas são evitáveis pela atenção à saúde, bem como, identificação de eventos sentinelas sensíveis à qualidade da atenção. As pesquisas são relevantes por trazerem objetividade, oportunidade, facilidade e disponibilidade dos indicadores de mortes evitáveis, contribuindo, entre outros aspectos, para análises de suas tendências temporais e comparações de suas probabilidades estimadas entre regiões e municípios (Malta et al., 2007).

A análise de óbitos infantis e fetais tem feito uso de algumas classificações de evitabilidade, conforme a viabilidade preventiva, com maior ênfase aos óbitos cuja origem esteja em problemas potencialmente tratáveis. Com isso, busca-se ter um maior esclarecimento e percepção, de forma sistematizada, do reflexo dos diferentes fatores que contribuem para a mortalidade infantil e fetal, e por conseguinte, adquire-se meios mais precisos de avaliar a efetividade dos serviços (Brasil, 2009b).

Em relação a óbitos evitáveis, o Sistema de Informação sobre Mortalidade (SIM) (Brasil, 2018), realizou um levantamento a nível nacional, considerando 10 anos (2007-2017). O documento expõe que na faixa etária de 0 a 27 dias, $75 \%$ dos óbitos são evitáveis, conforme tabela 01.

Tabela 01 - Distribuição das causas dos óbitos evitáveis segundo tipo de evitabilidade, por componente da mortalidade em neonatos (0 a 27 dias de nascido), 2007 a 2017. Brasil, 2021.

\begin{tabular}{|c|c|c|c|c|c|c|c|c|c|c|c|}
\hline \multirow[t]{2}{*}{ Variáveis } & \multicolumn{11}{|c|}{ Ano de ocorrência } \\
\hline & 2007 & 2008 & 2009 & 2010 & 2011 & 2012 & 2013 & 2014 & 2015 & 2016 & 2017 \\
\hline Imunização & 0,0 & 0,0 & 0,0 & 0,0 & 0,0 & 0,1 & 0,1 & 0,1 & 0,0 & 0,0 & 0,0 \\
\hline $\begin{array}{l}\text { Atenção à } \\
\text { mulher na } \\
\text { gestação }\end{array}$ & 45,5 & 46,1 & 45,6 & 47,3 & 47,5 & 47,2 & 48,7 & 48,9 & 47,6 & 48,1 & 48,4 \\
\hline $\begin{array}{c}\text { Atenção } \\
\text { durante o } \\
\text { trabalho de } \\
\text { parto e parto }\end{array}$ & 19,5 & 19,3 & 19,2 & 19,3 & 18,4 & 18,3 & 18,1 & 17,8 & 18,3 & 18,0 & 18,2 \\
\hline $\begin{array}{l}\text { Atenção ao } \\
\text { recém-nascido }\end{array}$ & 32,8 & 32,4 & 33,1 & 31,5 & 31,9 & 32,1 & 30,6 & 31,0 & 31,8 & 31,6 & 31,2 \\
\hline $\begin{array}{c}\text { Diagnóstico e } \\
\text { tratamento } \\
\text { adequado }\end{array}$ & 0,9 & 1,0 & 0,8 & 0,8 & 0,9 & 0,8 & 0,9 & 0,8 & 0,8 & 0,8 & 0,7 \\
\hline $\begin{array}{l}\text { Promoção à } \\
\text { saúde } \\
\text { vinculada a } \\
\text { ações de } \\
\text { atenção }\end{array}$ & 1,3 & 1,2 & 1,2 & 1,1 & 1,3 & 1,6 & 1,7 & 1,4 & 1,4 & 1,5 & 1,4 \\
\hline
\end{tabular}

Fonte: Sistema de Informações sobre Mortalidade.

Além disso, considerando-se a idade entre 28 dias e 04 anos, tem-se que $49 \%$ de mortes são evitáveis, como demonstra-se na tabela 2 . 
Tabela 02- Distribuição das causas dos óbitos evitáveis segundo tipo de evitabilidade, por componente da mortalidade infantil (28 dias a 4 anos de nascido), 2007 a 2017. Brasil, 2021.

\begin{tabular}{|c|c|c|c|c|c|c|c|c|c|c|c|}
\hline \multirow[t]{2}{*}{ Variáveis } & \multicolumn{11}{|c|}{ Ano de ocorrência } \\
\hline & 2007 & 2008 & 2009 & 2010 & 2011 & 2012 & 2013 & 2014 & 2015 & 2016 & 2017 \\
\hline Imunização & 0,3 & 0,4 & 0,2 & 0,4 & 0,6 & 1,0 & 1,0 & 1,6 & 0,6 & 0,3 & 0,5 \\
\hline $\begin{array}{c}\text { Atenção à } \\
\text { mulher na } \\
\text { gestação }\end{array}$ & 5,3 & 5,8 & 5,8 & 6.9 & 6,3 & 7,0 & 7,5 & 7,8 & 8,2 & 7,7 & 8,0 \\
\hline $\begin{array}{c}\text { Atenção } \\
\text { durante o } \\
\text { trabalho de } \\
\text { parto e parto }\end{array}$ & 2,7 & 2,6 & 2,6 & 2,7 & 2,7 & 2,3 & 2,4 & 2,6 & 2,4 & 2,7 & 2,6 \\
\hline $\begin{array}{c}\text { Atenção ao } \\
\text { recém- } \\
\text { nascido }\end{array}$ & 8,3 & 8,6 & 9,2 & 8,3 & 9,1 & 8,7 & 8,2 & 8,8 & 9,6 & 9,3 & 10,9 \\
\hline $\begin{array}{c}\text { Diagnóstico e } \\
\text { tratamento } \\
\text { adequado }\end{array}$ & 41,4 & 41,2 & 42,3 & 41,5 & 43,7 & 43,0 & 42,4 & 41,7 & 40,7 & 40,9 & 40,0 \\
\hline $\begin{array}{l}\text { Promoção à } \\
\text { saúde } \\
\text { vinculada a } \\
\text { ações de } \\
\text { atenção }\end{array}$ & 42,0 & 41,5 & 40,0 & 40,3 & 37,6 & 38,0 & 38,5 & 37,6 & 38,4 & 39,1 & 38,1 \\
\hline
\end{tabular}

Perante ao exposto é importante refletir acerca dos princípios do Sistema Único de Saúde, a saber: i) universalidade (todos devem ter acesso aos serviços de saúde), ii) integralidade (atendimento em todos os níveis de atenção à saúde considerando o ser humano como um todo), iii) equidade (princípio de justiça, pelo qual deve-se ofertar mais cuidados àqueles que mais necessitam) e intersetorialidade (assistência em saúde corroborada com a assistência de outros setores como social, educacional, de justiça e outros)(Brasil, 1990; Carmo; Guizardi, 2017) que, ao serem implementados, permitiriam a identificação precoce das causas da mortalidade infantil, estando estas relacionadas à gestação, parto e/ou pós-parto.

Neste contexto, destacam-se as atividades realizadas pelos comitês de prevenção do óbito infantil e fetal, que são instrumentos de gestão que permitem avaliar a qualidade da assistência à saúde prestada à gestante e à criança no primeiro ano de vida, para subsidiar as políticas públicas e as ações de intervenção, permitindo a visibilização necessária para enfrentamento do problema através do acompanhamento e monitoramento desses óbitos (Brasil, 2009a).

De fato, uma importante estratégia adotada para alcançar as metas pactuadas para redução da mortalidade infantil é a investigação de óbitos, realizada por meio dos Comitês de Prevenção do Óbito Infantil e Fetal, regulamentados pela portaria 1.399 de 1999, que se apresentam como um compromisso do Governo Federal em traçar ações para a redução da mortalidade infantil evitável com estruturação e aprimoramento das atividades desenvolvidas (Brasil, 2004). Os comitês são responsáveis pela investigação, análise dos óbitos, qualificação da informação, definição de medidas preventivas, bem como, divulgação e educação profissional e populacional (Brasil, 2004).

Venâncio e Paiva (2010) apontam que a atuação plena destes órgãos está condicionada à sua implantação ampla, com concepção clara, infraestrutura adequada e, principalmente, ser tida como um pólo técnico/gerencial, cujas ações ou recomendações tenham o respaldo das autoridades. No entanto, 
apesar das recomendações, os comitês ancoram-se em uma cultura de não obrigatoriedade, enfrentando dessa forma, uma carência ampla de recursos para o seu funcionamento, cujo suporte vem muitas vezes, do trabalho voluntário de servidores, caracterizando uma ausência de institucionalidade dos comitês, requerendo assim, uma ênfase necessária ao reconhecimento da importância da atuação de autoridades (gestores públicos) (Venâncio\&Paiva, 2010).

Apesar das dificuldades, diversas experiências no mundo mostram a importância dos comitês de mortalidade e investigação de óbitos como um recurso significativo para que se possa compreender melhor o óbito e suas circunstâncias, promovendo condições de identificar, com mais clareza, os fatores de risco e, por conseguinte, possibilitar uma reorientação das políticas de saúde voltadas para a redução da mortalidade infantil e materna (Brasil, 2009a). Ainda, Mathias (2009) esclarece que os comitês, diante de óbito infantil, passam a desenvolver estudos de casos com o objetivo de investigar os fatores de sua ocorrência.

Para Bernardino et al., (2015), que traçam em seu estudo a correlação entre mortalidade infantil e desigualdades sociais, a necessidade de conhecimento do contexto em que o óbito ocorre aparece claramente como uma forma viável de pensar a redução das iniquidades e injustiças sociais, de forma que os comitês incluam em suas ações aspectos biológicos, socioeconômicos e de acesso aos serviços, reconhecidos como determinantes da mortalidade infantil e fetal.

Ferreira et al., (2014) corroboram com esse pensamento, analisando que diante da constatação de que há essa heterogeneidade perceptível no perfil da população entre as regiões, geralmente marcando um quadro de desigualdades e levando em consideração as circunstâncias maternas em que esses óbitos infantis ocorrem, a compreensão da distribuição dos fatores de risco sociais se faz fundamental. Da mesma forma, deve-se procurar compreender como esses fatores variam conforme cada região e sobre o perfil da morbimortalidade perinatal que está intrinsecamente relacionada à qualidade da assistência, potencializando-se a possibilidade de avaliar a qualidade dos serviços de saúde.

Embora o conhecimento dos fatores e causas associadas os óbitos infantis seja importante para atuação dos comitês, é importante citar um problema ainda persistente, de acordo com Santos (2012), que é o sub-registro de óbitos, comuns principalmente nas regiões Norte e Nordeste do Brasil. Ainda existe uma omissão desses registros em cartório, tanto pela impossibilidade ou burocracia no acesso aos registros, como pela existência de cemitérios irregulares, aliado ao desconhecimento da população sobre a importância da declaração de óbito, que inviabiliza o registro formal (Santos, 2012).

Este trata-se de um problema a mais que exige um enfrentamento por parte dos comitês, uma vez que a subnotificação repercute em ações adequadas de saúde para a diminuição dos índices. Junta-se ainda outra questão, que é a baixa qualidade das informações nas declarações de óbito, representada pelo grande contingente de causas mal definidas - imprecisões na declaração da "causa da morte" - e campos não preenchidos, dificultando a análise dos fatores que influenciam a mortalidade e consequentemente, dificultando as ações de intervenção (Santos, 2012).

Na pesquisa de Araújo (2012), cujo objetivo foi analisar a implantação e operacionalização dos Comitês de Prevenção do Óbito Infantil e Fetal no Estado do Ceará, abrangendo 18 municípios cearenses, foi constatada deficiência na realização da vigilância do óbito infantil. Segundo a pesquisa, o período de tempo decorrido entre a data do óbito e o acesso pelos comitês às informações sobre o óbito equivale a mais de um mês em 15 (88\%) comitês. Ainda, foi observado como maior desafio, as dificuldades para o cumprimento dos prazos, cuja justificativa seria a demora das instituições notificantes do óbito na alimentação do sistema de informação.

Os comitês utilizam tanto as fichas padronizadas para investigação do óbito infantil e fetal do Ministério da Saúde, como também, outros documentos, como prontuário hospitalar e ambulatorial, ficha da gestante, cartão da criança e gestante, formulário de entrevista domiciliar, declarações de 
nascidos vivos e óbito, fichas de atendimento, receitas médicas e laudos de necropsia (Araújo, 2012). São citados outros documentos como os relatórios da central de regulação, guias de sepultamento e listagem dos cartórios. Entre os documentos que os comitês têm dificuldade de acesso para realizar a investigação do óbito infantil e fetal registram-se o acesso ao prontuário hospitalar, fichas de encaminhamento e laudos de necropsia (Araújo, 2012). O acesso a esses documentos e sua devida análise apontarão causas e as classificarão como evitáveis ou não.

Assim, reitera-se o que vem corroborado o estudo de Brandão, Goteiro e Monteiro (2012), quando citam o quão frequentemente ocorre casos de óbito infantil que são potencialmente evitáveis, podendo-se estimar em torno de mais da metade dos casos. Isso o reforça a importância de que esses óbitos sejam analisados pelos comitês, priorizando o enfoque da evitabilidade, permitindo fornecer um maior esclarecimento e percepção de como distintos fatores podem ser utilizados para que se trabalhe a redução da mortalidade infantil e fetal (Brandão, Goteiro \& Monteiro, 2012).

Ruoff, Andrade e Piccolo (2018) chamam ainda a atenção para a necessidade de que as pessoas difundam mais o processo de análise da evitabilidade dos óbitos realizado pelos comitês, o que não costuma ocorrer com frequência. Para as autoras, é preciso buscar uma exploração maior desse processo, cuja compreensão é fundamental para que se possa evidenciar a forma como as atividades dos comitês podem contribuir para a redução da mortalidade, e, dessa forma, terem sua importância reconhecida e sua obrigatoriedade exigida.

Sob uma perspectiva voltada para a evitabilidade, a análise do óbito ocorre mediante informações como o percurso da gestante e da criança dentro da rede de atenção como também, nos três níveis de atenção à saúde, levando em conta os fatores biológicos, familiar e socioeconômico (Ruoff, Andrade \&Picoli, 2018). Analisa-se ainda, se os serviços de saúde, através do suporte da rede de atenção à saúde, têm funcionado de forma resolutiva, assim como o acesso à assistência de baixa e alta tecnologia, condições das instituições, acesso ao tratamento e constituição e processo de trabalho das equipes multidisciplinares, presentes nas redes de atenção (Ruoff, Andrade \& Piccolo, 2018).

É preciso levar em consideração que essas dificuldades devem ser sanadas, mesmo que o quadro descrito acima não caracterize a realidade de todos os comitês no Brasil, mas, alertam para a necessidade de que haja o pensamento que a redução da mortalidade infantil e fetal terá suas medidas consolidadas a partir da efetivação das ações dos comitês e, para isso, a mobilização de parceiros é fundamental, envolvendo, principalmente, àqueles que estão em postos hierárquicos de poder, como os gestores públicos.

\section{Considerações Finais}

Diante dos achados na literatura, observa-se que existem vários determinantes para uma atuação sólida e assertiva dos comitês, entre eles, a concretização de políticas públicas que minimizem as iniquidades sociais, sendo fundamental para a atuação desses comitês, a análise regional da assistência em saúde direcionada ao público materno e infantil, que inclui a atenção à gestante e principalmente, às crianças, contribuindo para a redução da mortalidade infantil e fetal. 
Vale refletir sobre os determinantes socioeconômicos mediante o atual cenário de crise econômica e desemprego, em que a população pobre se encontra em maior vulnerabilidade, podendo repercutir diretamente no aumento de óbitos infantis pela escassez de direitos básicos à qualidade da gestação, parto e vida pós parto. Nesse sentido, não somente políticas sociais que sanem tal escassez devem ser implementadas e fiscalizadas, mas também deve-se incentivar a criação e institucionalização de comitês capacitados.

Além disso, é importante desconstruir a cultura da maternidade compulsória que além de colocar o papel de mãe como obrigatório à mulher, naturalizando a maternidade sob qualquer custo, até mesmo a partir de uma assistência desumanizada e de má qualidade; ainda incita a mulher a compreender que o cuidado lhe é inerente, não percebendo, dessa forma, a necessidade da educação em saúde ou de políticas públicas que fortaleçam a qualidade de vida da díade mãe-bebê.

A informação adequada, oportuna e em tempo hábil para um mapeamento adequado do quadro situacional é outro fator importante e que parece ainda se constituir em lacuna significativa para atuação dos comitês. Além disso, observou-se um índice ainda alto de mortalidade infantil por causas evitáveis.

Considera-se a produção deste estudo importante, por contribuir para uma reflexão e maior disseminação de conhecimentos das ações e da importância dos comitês, sendo que se pode apontar como limitação, a necessidade de estudos de campo sobre a temática, que revelassem realidades concretas sobre a atuação dos comitês, pois ainda há uma escassez neste sentido.

Em suma, há um quadro ainda preocupante frente à mortalidade infantil e fetal no Brasil, mas que pode encontrar na implantação dos Comitês de Mortalidade Infantil e Fetal, uma evolução resolutiva. Para isso, é necessário comprometimento e mobilização político-administrativa, em que se possa realmente ter uma redução dos indicadores de mortalidade infantil que não se restrinja apenas a superar índices anteriores, mas de superar metas e arbitrando, com equidade, as tensões sociais. 


\section{Referências Bibliográficas}

Andrade, Carla., \& Szwarcwald, Célia. (2007). Desigualdades sócio-espaciais da adequação das informações de nascimentos e óbitos do Ministério da Saúde, Brasil, 2000-2002. Cadernos de saúde pública, 23(5), 1207-1216. https://doi.org/10.1590/S0102-311X2007000500022

Araújo, L. B. (2012). Análise da Implantação e Operacionalização dos Comitês de Prevenção do Óbito Infantil e Fetal no Estado do Ceará. 2012. 97f. Dissertação (Mestrado). Universidade Estadual do Ceará, Fortaleza, Brasil. Acessado em 04 de Março de 2020, de: https://bdtd.ibict.br/vufind/Record/UECE-0 6b5104c11c5d3c751669fb8f1363a6df

Bernardino, Lilia., Costa, Simone., Lima, Cassio., Brito, Maria., Dias, Orlene., \& Freitas, Daniel. (2015). Mortalidade infantil e desigualdade social: análise fundamentada na bioética de intervenção. Revista Renome, 4(2), 42-60. Acessado em 04 de Março de 2020, de: https://www.periodicos.unimontes.br/index.php/renome/article/view/2689

Brandão, Isabel., Godeiro, Ana., \& Monteiro, Akemi. (2012). Assistência de enfermagem no pré-natal e evitabilidade de óbitos neonatais. Revista Enfermagem UERJ, 20(5), 596-602. Acessado em 04 de Março de 2020, de: publicacoes.uerj.br/index.php/enfermagemuerj/article/view/5807/4228

Brasil. (2000). Atenção Integrada às Doenças Prevalentes na Infância. Brasília: Ministério da Saúde. Acessado em 04 de Março de 2020, de: http://www.saúde.gov.br/programas/scriança/criança/aidpi.htm

Brasil. (2004). Manual dos comitês de prevenção do óbito infantil e fetal. Secretaria de Atenção à Saúde. Departamento de Ações Programáticas Estratégicas. Brasília: Ministério da Saúde.

Brasil. (2009a). Nota técnica: Vigilância epidemiológica de óbitos infantis e fetais. Secretaria de Vigilância em Saúde. Departamento de análise de situação de saúde. Coordenação geral de informações e análise epidemiológica. Brasília: Ministério da Saúde.

Brasil. (2009b). Manual de vigilância do óbito infantil e fetal e do comitê de prevenção do óbito infantil e fetal. (Série A. Normas e Manuais Técnicos). Brasília: Editora do Ministério da Saúde.

Brasil. (2010, 11 de jan.). Portaria $n^{o} 72$. Dispõe sobre a regulamentação da Vigilância de Óbitos Infantis e Fetais. Diário Oficial daUnião, 2010.

Brasil. (2018). Evolução da mortalidade na infância nos últimos 10 anos (2007 a 2016). Brasília: Secretaria de Vigilância à Saúde.

Brasil. (1990, 19 de Set.). Lei $n^{\circ}$ 8.080, Lei Orgânica da Saúde. Dispõe sobre as condições para a promoção, proteção e recuperação da saúde, a organização e o funcionamento dos serviços correspondentes e dá outras providências. Brasília.

Carmo, Michelly., \& Guizardi, Francini. (2017). Desafios da intersetorialidade nas políticas públicas de saúde e assistência social: uma revisão do estado da arte. Physis: Revista de Saúde Coletiva, 27(4), 1265-1286. Acessado em 04 de Março de 2020, de: https://doi.org/10.1590/S0103$\underline{73312017000400021}$

Domingues, Rosa.., Hartz, Zulmira., Dias, Marcos., \& Leal, Maria. (2012). Avaliação da adequação da assistência pré-natal na rede SUS do Município do Rio de Janeiro, Brasil. Cadernos de Saúde Pública, 28(3), 425-437. Acessado em 04 de Março de 2020, de: https://doi.org/10.1590/S0102$\underline{311 X 2012000300003}$

Elias, Cláudia., Silva, Leandro., Martins, Mirian., Ramos, Neide., Souza, Maria., \& Hipólito, Rodrigo. (2012). Quando chega o fim? Uma revisão narrativa sobre terminalidade do período escolar para 
alunos deficientes mentais. SMAD Revista Eletrônica Saúde Mental Álcool e Drogas, 8(1), 48-53. Acessado em 04 de Março de 2020, de: https://doi.org/10.11606/issn.1806-6976.v8i1p48-53

Ferreira, Ana., Soares, Viviane., Nitschke, Rosane., Tholl, Adriana., Muñoz, Maria., \& Michelin, Samanta. (2014). O quotidiano de gestantes: a enfermagem promovendo o ser saudável. Texto \& Contexto-Enfermagem, 23(4), 987-994. Acessado em 04 de Março de 2020, de: https://doi.org/10.1590/0104-07072014001110012

Fertonani, Hosanna., Pires, Denise., Biff, Daiana., \& Scherer, Magda. (2015). Modelo assistencial em saúde: conceitos e desafios para a atenção básica brasileira. Ciência \& Saúde Coletiva, 20(6), Acessado em 04 de Março de 2020, de: https://doi.org/10.1590/1413-81232015206.13272014.

Freitas, Aline. S. (2019). Desenho Jurídico-Institucional da Valorização do Salário Mínimo no Brasil dos Governos Dilma ao Governo Bolsonaro: quais os elementos jurídicos por trás dos discursos políticos?. Revista Gestão \& Políticas Públicas, 9(1), 32-50. Acessado em 04 de Março de 2020, de: https://doi.org/10.11606/rgpp.v10i1.174586

Frias, Paulo., \& Navarro, Leila. (2013). Crianças: sujeitos de direito e sua vulnerabilidade. Em Sonia Bittencourt., Marcos Dias., \& Mayumi Wakimoto. (Orgs). Vigilância do óbito materno, infantil e fetal e atuação em comitês de mortalidade. Rio de Janeiro: Fiocruz, 91-133.

Galvão, Lize B. (2020). Mãe solteira não. Mãe solo! Considerações sobre maternidade, conjugalidade e sobrecarga feminina. Revista Direito e Sexualidade, 1(1), 1-23. Acessado em 04 de Março de 2020, de: https://periodicos.ufba.br/index.php/revdirsex/article/view/36872/21118

Garcia, Carla Cristina. (2019). Notas sobre a história dos trabalhos das mulheres na sociedade ocidental: das diferenças as desigualdades laborais de gênero. Revista Gestão e Políticas Públicas, 9(1): 123-140. Acessado em 04 de Março de 2020, de: https://www.revistas.usp.br/rgpp/article/view/175097

IBGE. (2019). Tábua completa de mortalidade para o Brasil - 2018: Breve análise da evolução da mortalidade no Brasil. Rio de Janeiro: Ministério da Economia, 2019.

Klein, Cecília de Jesus., Madi, José Mauro., Araújo, Breno Fauth de., Zatti, Helen., Dal Bosco, Deize dos Santos., Henke, Claudia Nicole., Rombaldi, Renato Luís., Madi, Sônia Regina Cabral. (2012). Fatores de risco relacionados à mortalidade fetal. Revista da AMRIGS, 56(1), 11-16.

Lisboa, Luiza., Abreu, Daisy., Lana, Ângela., \& França, Eelisabeth. (2015). Mortalidade infantil: principais causas evitáveis na região Centro de Minas Gerais, Brasil, 1999-2011. Epidemiologia e Serviços de Saúde, 24, 711-720. Acessado em 04 de Março de 2020, de: https://www.scielo.br/pdf/ress/v24n4/2237-9622-ress-24-04-00711.pdf

López-Barreyro, Luz A. (2017). A Imagem da Mulher nas Propagandas Televisivas: uma análise na perspectiva de Gênero. Revista Gestão \& Políticas Públicas, 7(1), 37-56. Acessado em 04 de Março de 2020, de: https://www.revistas.usp.br/rgpp/article/view/137724

Malta, Deborah Carvalho, Duarte, Elisabeth Carmen, Almeida, Márcia Furquim de, Dias, Maria Angélica de Salles, Morais Neto, Otaliba Libânio de, Moura, Lenildo de, Ferraz, Walter, \& Souza, Maria de Fatima Marinho de. (2007). Lista de causas de mortes evitáveis por intervenções do Sistema Único de Saúde do Brasil. Epidemiologia e Serviços de Saúde, 16(4), 233-244. Acessado em 04 de Março de 2020, de: https://dx.doi.org/10.5123/S1679-49742007000400002

Mario, Débora., Rigo, Lilian., Boclin, Karine., Malvestio, Lygia., Anziliero, Deniz., Horta, Bernando., Wehrmeister, Fernando., \& Martínez-Mesa, Jeovany. (2019). Qualidade do Pré-Natal no Brasil: Pesquisa Nacional de Saúde 2013. Ciência \& Saúde Coletiva, 24(3), 1223-1232. Acessado em 04 de Março de 2020, de: https://doi.org/10.1590/1413-81232018243.13122017

Martins, Eunice Francisca, Rezende, Edna Maria, Lana, Francisco Carlos Félix, \& Souza, Kleyde Ventura de. (2013). Óbitos perinatais investigados e falhas na assistência hospitalar ao 
parto. Escola Anna Nery, 17(1), 38-45. Acessado em 04 de Março de 2020, de: https://doi.org/10.1590/S1414-81452013000100006

Mathias, Thais., Uchimura, Taqueco., Assunção, Amanda., \& Predebon, Kelen. (2009). Atividades de extensão universitária em comitê de prevenção de mortalidade infantil e estatísticas de saúde. Revista Brasileira de Enfermagem, 62(2), 205-311. Acessado em 04 de Março de 2020, de: https://www.scielo.br/scielo.php?script=sci_abstract\&pid=S0034$71672009000200022 \& \operatorname{lng}=\mathrm{pt} \& \mathrm{nrm}=\mathrm{iso} \& \mathrm{t} \operatorname{lng}=\mathrm{pt}$

Muñoz, Luz., Sanchez, Ximena., Arcos, Estela., Vollrath, Antonia., \& Bonatti, Carla. (2013). Vivenciando a maternidade em contextos de vulnerabilidade social: uma abordagem compreensiva da fenomenologia social. Revista Latino-Americana de Enfermagem, 21(4), 913-919. Acessado em 04 de Março de 2020, de: https://www.scielo.br/j/rlae/a/mKR3r9RkPZJJBVMVJ5LFSxP/?format=pdf\&lang=pt

Ruoff, Andriela., Andrade, Selma., \& Piccoli, Talita. (2018). O processo de análise da evitabilidade dos casos de óbito infantil e fetal: estudo de caso único. Texto \& Contexto-Enfermagem, 27(4). Acessado em 04 de Março de 2020, de: https://www.scielo.br/pdf/tce/v27n4/0104-0707-tce-27-04e4030017.pdf

Santos, Antonio e cols. (2012). Estimativas da mortalidade infantil: uma adaptação do estimador bayesiano às microrregiões do estado do Rio Grande do Norte (2000-2009). Em XVIII Encontro Nacional de Estudos Populacionais. Águas de Lindóia, SP.

Silva, Joise., Marques, Patrícia. \& Paiva, Mirian. (2013). Saúde sexual e reprodutiva e enfermagem: um pouco de história na Bahia. Rev. bras. enferm. 66(4), 501-507.

Silva, Vitória., Tavares, Nicole., Silva, Milena., Silva, Izabela., Rêgo, Talita., Silva, Douglas e cols. (2019). Fatores associados ao óbito fetal na gestação de alto risco: Assistência de enfermagem no pré-natal. Revista Eletrônica Acervo Saúde, 37, e1884-e1884. Acessado em 04 de Março de 2020, de: https://doi.org/10.25248/reas.e1884.2019

Tomasi, Eliane., Fernandes, Pedro., Fischer, Tatlita., Siqueira, Fernando., Silveira, Denise., Thumé, Elaine., Duro, Suele., Saes, Mirele., Nunes. Bruno., Fassa, Anaclaudia., \& Facchini, L. A. (2017). Qualidade da atenção pré-natal na rede básica de saúde do Brasil: indicadores e desigualdades sociais. Cadernos de saúde pública, 33(3), e00195815. Acessado em 04 de Março de 2020, de: https://doi.org/10.1590/0102-311X00195815

Venâncio, Sônia I., \& Paiva, Rui D. (2010). O processo de implantação dos Comitês de Investigação do Óbito Infantil no Estado de São Paulo. Revista Brasileira de Saúde Materno Infantil, 10(3), 369-375. Acessado em 04 de Março de 2020, de: https://www.scielo.br/scielo.php?script=sci_arttext\&pid=S1519-38292010000300010 